\title{
Deaths in children and young people in England after SARS-CoV-2 infection during the first pandemic year
}

\author{
Clare Smith $\mathbb{1}^{1,2} \bowtie$, David Odd $\mathbb{1}^{3,4}$, Rachel Harwood ${ }^{5,6}$, Joseph Ward ${ }^{7}$, Mike Linney ${ }^{8,9}$, \\ Matthew Clark ${ }^{1}{ }^{1}$, Dougal Hargreaves ${ }^{10}{ }^{10}$, Shamez N. Ladhani ${ }^{11,12}$, Elizabeth Draper ${ }^{13}$,

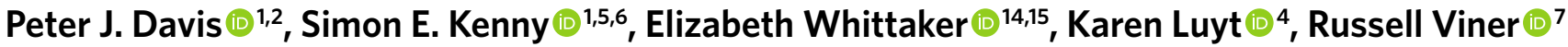 \\ and Lorna K. Fraser ${ }^{10} 16$
}

Severe acute respiratory syndrome coronavirus 2 (SARS-CoV-2) infection is rarely fatal in children and young people (CYP, $<\mathbf{1 8}$ years old), but quantifying the risk of death is challenging because CYP are often infected with SARS-CoV-2 exhibiting no or minimal symptoms. To distinguish between CYP who died as a result of SARS-CoV-2 infection and those who died of another cause but were coincidentally infected with the virus, we undertook a clinical review of all CYP deaths with a positive SARS-CoV-2 test from March 2020 to February 2021. The predominant SARS-CoV-2 variants were wild-type and Alpha. Here we show that, of 12,023,568 CYP living in England, 3,105 died, including 61 who were positive for SARS-CoV-2. Of these deaths, 25 were due to SARS-CoV-2 infection (mortality rate, two per million), including 22 due to coronavirus disease 2019-the clinical disease associated with SARS-CoV-2 infection-and 3 were due to pediatric inflammatory multisystem syndrome temporally associated with SARS-CoV-2. In total, $99.995 \%$ of CYP with a positive SARS-CoV-2 test survived. CYP older than 10 years, Asian and Black ethnic backgrounds and comorbidities were over-represented in SARS-CoV-2-related deaths compared with other CYP deaths. These results are important for guiding decisions on shielding and vaccinating children. New variants might have different mortality risks and should be evaluated in a similar way.

dentifying CYP at risk of severe illness and death after SARS-CoV-2 infection is essential to guide families, clinicians and policymakers about future shielding policies, school attendance, novel therapeutic agents and vaccine prioritization.

SARS-CoV-2 infection is usually mild and asymptomatic in $\mathrm{CYP}^{1-3}$. Therefore, CYP have comprised a very low proportion of all hospitalizations and deaths from coronavirus disease 2019 (COVID-19) globally ${ }^{4}$. The clinical manifestations of COVID-19 in CYP are different than those in adults ${ }^{1}$. Although many CYP present with the typical fever, cough and shortness of breath, they also present with broader non-specific symptoms, including abdominal pain, nausea, headache and sore throat ${ }^{1,3}$. This, in combination with a mild or asymptomatic phenotype ${ }^{2}$, provides a challenge for describing how SARS-CoV-2 directly affects CYP.

Severe illness and death associated with SARS-CoV-2 in CYP is rare and can be due to either acute COVID-19 or pediatric inflammatory multisystem syndrome temporally associated with SARS-CoV-2 (PIMS-TS) ${ }^{2,5}$. PIMS-TS, also called multisystem inflammatory syndrome in children, is a rare syndrome characterized by persistent fever, inflammation (neutrophilia, lymphopenia and raised C-reactive protein) and evidence of single- or multi-organ dysfunction that might occur concurrently or after infection ${ }^{6}$. As death from acute COVID-19 or PIMS-TS in CYP is extremely rare $^{4,7,8}$, those who have died have been poorly characterized ${ }^{9}$. Furthermore, it remains unclear to what extent these rare deaths relate directly to the pathological processes of COVID-19 or whether CYP who died from alternative causes were coincidentally positive for SARS-CoV-2 around the time of death. This issue is made more difficult by the very high prevalence of asymptomatic infection at times of high prevalence, with reported prevalence up to $4-6 \%$ of United Kingdom CYP during December 2020 (ref. ${ }^{10}$ ). The distinction between those who died of SARS-CoV-2 infection and those who died of an alternative cause with a coincidental positive SARS-CoV-2 test is important for understanding which CYP are truly at higher risk for severe disease or death.

Answering this important question required detailed examination of all deaths in a large population, going beyond simple cause of death registration, to review the contribution of SARS-CoV-2 to death. We used detailed clinical data in the National Child Mortality Database $(\mathrm{NCMD})^{11}$, which is a comprehensive and unique mandatory

'NHS England and NHS Improvement, London, UK. 2Paediatric Intensive Care Unit, Bristol Royal Hospital for Children, University Hospitals Bristol and Weston NHS Foundation Trust, Bristol, UK. ${ }^{3}$ Division of Population Medicine, Cardiff University, Cardiff, Wales, UK. ${ }^{4}$ National Child Mortality Database, University of Bristol, Bristol, UK. ${ }^{5}$ Institute of Systems, Molecular and Integrative Biology, University of Liverpool, Liverpool, UK. ${ }^{6}$ Department of Paediatric Surgery, Alder Hey in the Park, Liverpool, UK. 'Population, Policy and Practice Department, UCL Great Ormond St. Institute of Child Health, London, UK. ${ }^{8}$ Royal College of Paediatrics and Child Health, London, UK. ' Department of Paediatrics, University Hospitals Sussex NHS Foundation Trust, Brighton, UK. ${ }^{10} \mathrm{~S}$ chool of Public Health, Imperial College London, London, UK. "Immunisation and Countermeasures, Public Health England, London, UK. ${ }^{12}$ Paediatric Infectious Diseases Research Group, St. George's University of London, London, UK. ${ }^{13}$ Paediatric Intensive Care Audit Network (PICANet), Department of Health Sciences, University of Leicester, Leicester, UK. ${ }^{14}$ Department of Paediatric Infectious Diseases, Imperial College Healthcare NHS Trust, London, UK. ${ }^{15}$ Section of Paediatric Infectious Diseases, Faculty of Medicine, Imperial College London, London, UK. ${ }^{16}$ Martin House Research Centre, University of York, York, UK.凶e-mail: clare.smith107@nhs.net 


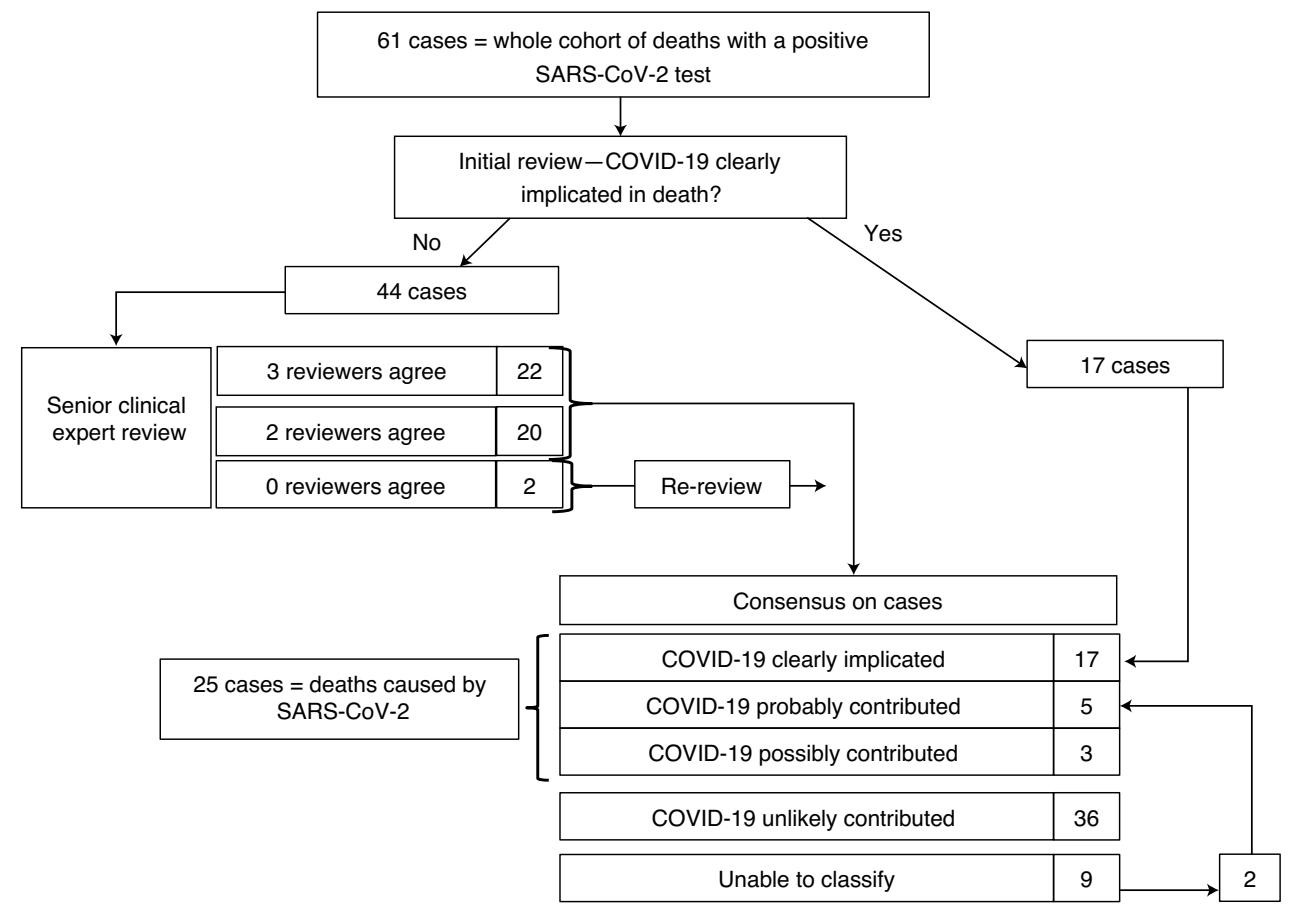

Fig. 1 | Flow diagram of the approach used to determine if SARS-CoV-2 contributed to death or if it was a coincidental finding. This approach was applied to all CYP who died and had a positive SARS-CoV-2 test. The numbers are included at each representative stage.

national dataset of deaths of individuals younger than 18 years of age, to review the contribution of SARS-CoV-2 to death.

If higher-risk groups are identified, they might benefit from vaccination and/or protective 'shielding' at times of high prevalence, whereas 'shielding' based upon erroneous assumptions of vulnerability is likely to cause significant secondary harms-for example, the effects of not attending school and restrictive or reduced socializing, affecting both development and mental health. Similarly, risks from the disease need to be weighed against potential risks of vaccination in informing vaccination policy. Therefore, this study aimed to:

1. Quantify the number of CYP who died of SARS-CoV-2 by differentiating between CYP who died of SARS-CoV-2 and those who died of an alternative cause with a coincidental positive SARS-CoV-2 test.

2. Assess the clinical and demographic characteristics of the CYP who died of SARS-CoV-2 compared to CYP deaths from all other causes during the first pandemic year.

\section{Results}

Between March 2020 and February 2021, 3,105 CYP in England died of all causes. Of these, $61 \mathrm{CYP}$ had a positive SARS-CoV-2 test, and 3,044 died of all other causes.

Clinical records of the 61 CYP who died with a positive SARS-CoV-2 test were reviewed to identify if SARS-CoV-2 contributed to death. This process initially included identifying whether SARS-CoV-2 was listed as 1a (the direct cause of death) on the Certificate of Cause of Death and whether the clinical course described was typical of SARS-CoV-2 infection. In these circumstances, the classification 'SARS-CoV-2 clearly contributed to death' was applied. In England, the Certificate of Cause of Death is set out in two parts ${ }^{12}$. Part 1a is the immediate, direct cause of death. The sequence of events or conditions that led to the death are then listed as $1 \mathrm{~b}$ and $1 \mathrm{c}$ (if necessary) ${ }^{12}$.

If the role of SARS-CoV-2 in contributing to death was not clearly apparent, each case underwent review by three independent senior clinical experts in relevant fields (General Pediatrics, Neonatology and Pediatric Intensive Care) who were asked to classify each case. Definitions for each category and the details behind the process are outlined in the Supplementary Information and in Fig. 1.

In total, 25 (41\%) of the 61 CYP died of SARS-CoV-2, including 22 with acute COVID-19 and three with PIMS-TS. In the other 36 (59\%) of the 61 test-positive CYP, SARS-CoV-2 did not contribute to death (Table 1 and Figs. 1 and 2).

An estimated 469,982 CYP were infected with SARS-CoV-2 in England from March 2020 to February 2021, giving an infection fatality rate of five per 100,000 CYP $(0.005 \%)$ and, based on a population of $12,023,568$, a mortality rate of two per million CYP $(0.0002 \%)^{13}$.

Demographics. There were small amounts of missing demographic data for the reference population $(2.3 \%$ sex, $10.6 \%$ ethnicity and $0.6 \%$ deprivation (Table 1 ), but there were no missing demographic data for the 25 CYP who died of SARS-CoV-2.

CYP who died of SARS-CoV-2 $(n=25)$ were older than those who died from all other causes $(n=3,080)$ in the same time period. Eighteen of 25 (72\%) young people who died of SARS-CoV-2 were aged 10 years or older compared to $19 \%$ in the deaths from all other causes (chi-squared 59.7, $P<0.001$ ). All three deaths in CYP who died of PIMS-TS were aged 10-14 years. Of interest, specific to vaccination policy in the United Kingdom, there were eight deaths in young people aged $12-15$ years. The sex distribution was equally split between males and females (12 (48\%) and 13 (52\%), respectively) and did not differ from the deaths from all other causes (chi-squared $0.64, P=0.28$ ). A greater proportion of CYP from Asian (36\% compared to $16 \%)$ and Black (20\% compared to $8 \%)$ ethnicity died of SARS-CoV-2 compared to deaths from all other causes (chi-squared $17.9, P<0.001)$. The three CYP who died of PIMS-TS were from different ethnic groups. No significant difference was observed in the deprivation categories between CYP who died of SARS-CoV-2 and deaths from all other causes (chi-squared 0.35, $P=0.99$ ), although more CYP from more deprived areas died in both groups.

The mortality rate in CYP who died of SARS-CoV-2 was 0.2 per 100,000 (95\% confidence interval (CI), 0.1-0.3) compared to 
Table 1 | Demographic details for CYP who died between March 2020 and February 2021 from all causes and the 61 CYP who died with a positive SARS-CoV-2 test, split by the likely cause of death

\begin{tabular}{|c|c|c|c|c|c|c|c|c|c|}
\hline \multirow{4}{*}{ All deaths } & \multicolumn{8}{|c|}{ All deaths, March 2020-February $2021(n=3,105)$} & \multirow{4}{*}{$\begin{array}{l}\text { Comparison of } \\
\text { frequencies }^{\mathrm{a}}\end{array}$} \\
\hline & \multicolumn{5}{|c|}{ Reference population $(n=3,080)$, all other causes of death } & \multicolumn{3}{|c|}{ Died of COVID-19/PIMS-TS $(n=25)$} & \\
\hline & \multicolumn{2}{|c|}{$\begin{array}{l}\text { Died without positive } \\
\text { test for SARS-CoV-2 } \\
\qquad(n=3,044)\end{array}$} & \multicolumn{2}{|c|}{$\begin{array}{l}\text { Incidental positive } \\
\text { SARS-CoV-2 test at } \\
\text { death }(n=36)\end{array}$} & \multirow{2}{*}{$\begin{array}{c}\text { Est. rate per } \\
100,000 \text { person } \\
\text { years }(95 \% \\
\text { Poisson } \mathrm{CI})\end{array}$} & \multirow[t]{2}{*}{ Number } & \multirow[t]{2}{*}{ Percentage } & \multirow{2}{*}{$\begin{array}{c}\text { Est. rate per } \\
100,000 \text { person } \\
\text { years }(95 \% \\
\text { Poisson } \mathrm{Cl})\end{array}$} & \\
\hline & Number & Percentage & Number & Percentage & & & & & \\
\hline $0-27 d$ & 1.388 & $45.6 \%$ & 3 & $8.3 \%$ & $\begin{array}{l}289.3 \\
(276.8-302.1)\end{array}$ & 0 & $0.0 \%$ & $0.3(0.0-11.0)$ & $<0.001$ \\
\hline $28-364 d$ & 616 & $20.2 \%$ & 11 & $30.6 \%$ & & 2 & $8.0 \%$ & & \\
\hline $1-4$ years & 281 & $9.2 \%$ & 4 & $11.1 \%$ & $10.4(9.2-11.6)$ & 0 & $0.0 \%$ & $0.00(-)$ & \\
\hline $5-9$ years & 191 & $6.3 \%$ & 4 & $11.1 \%$ & $5.7(4.9-6.6)$ & 5 & $20.0 \%$ & $0.1(0.0-0.3)$ & \\
\hline Female & 1,667 & $54.8 \%$ & 18 & $50.0 \%$ & $22.4(21.2-23.7)$ & 12 & $48.0 \%$ & $0.2(0.1-0.4)$ & 0.28 \\
\hline Male & 1,308 & $43.0 \%$ & 18 & $50.0 \%$ & $27.1(25.9-28.5)$ & 13 & $52.0 \%$ & $0.2(0.1-0.3)$ & \\
\hline Missing & 69 & $2.3 \%$ & - & - & - & - & - & - & \\
\hline \multicolumn{10}{|l|}{ Ethnicity } \\
\hline $\begin{array}{l}\text { Asian or Asian } \\
\text { British }\end{array}$ & 471 & $15.5 \%$ & 10 & $27.8 \%$ & $31.3(28.6-34.2)$ & 9 & $36.0 \%$ & $0.6(0.3-1.1)$ & $<0.001$ \\
\hline $\begin{array}{l}\text { Black or Black } \\
\text { British }\end{array}$ & 241 & $7.9 \%$ & 4 & $11.1 \%$ & $37 \cdot 8(33 \cdot 2-42.9)$ & 5 & $20.0 \%$ & $0.8(0.3-1.8)$ & \\
\hline Mixed & 169 & $5.6 \%$ & 1 & $2.8 \%$ & $23.3(19 \cdot 9-27.1)$ & 4 & $16.0 \%$ & $0.5(0.1-1.4)$ & \\
\hline 4 & 402 & $13.2 \%$ & 3 & $8.3 \%$ & $18.7(16.9-20.6)$ & 3 & $12.0 \%$ & $0.1(0.0-0.4)$ & \\
\hline 5 & 337 & $11.1 \%$ & 4 & $11.1 \%$ & $15.3(13.8-17.1)$ & 2 & $8.0 \%$ & $0.1(0.0-0.3)$ & \\
\hline Missing & 18 & $0.6 \%$ & - & - & - & - & - & - & \\
\hline
\end{tabular}

Table 1 shows the demographic details for the CYP who died of all causes and the CYP who died of SARS-CoV-2. ${ }^{\text {aThe } C Y P}$ who died of SARS-CoV-2 were compared to CYP who died from all other causes using summary statistics, and differences between groups were compared using the two-sided chi-squared test or Fisher's exact test if small numbers. No adjustment for multiple testing was undertaken. Values are $n$ (\%) or median (interquartile range) as appropriate.

25.5 per 100,000 (95\% CI, 24.7-26.5) for all other causes of death. Although the proportion of CYP from Asian and Black ethnic groups who died of SARS-CoV-2 was higher, their absolute risk of death from SARS-CoV-2 was still extremely low at 0.6 per 100,000 (95\% CI, $0.3-1.1$ ) and 0.8 per 100,000 (95\% CI, 0.3-1.8), respectively. Similarly, the proportion of CYP aged 10-14 years and 15-17 years who died of SARS-CoV-2 was higher than the proportion of CYP in the same age categories who died of all other causes. However, their absolute risk of death from SARS-CoV-2 was still extremely low at 0.3 per 100,000 (95\% CI, $0.1-0.5)$ and 0.5 (95\% CI, $0.2-0.9)$ per 100,000 , respectively.

Comorbidities. A similar proportion of the 25 CYP who died of SARS-CoV-2 $(n=19,76 \%)$ and the 3,080 deaths from all other causes $(n=2,267,74 \%)$ (chi-squared $0.004, P=0.60$ ) had a chronic underlying health condition (Tables 2 and 3). Significantly more CYP who died of SARS-CoV-2 had a life-limiting condition $(n=15,60 \%)$ compared to deaths from all other causes $(n=988$, $32 \%$ ) (chi-squared 8.5, $P=0.005$ ). Of the 25 CYP who died of SARS-CoV-2, 64\% $(n=16)$ had comorbidities in two or more body systems compared to $45 \%(n=1,373)$ of the CYP who died from all other causes (chi-squared 5.5, $P=0.14$ ).

Six (24\%) of the 25 CYP who died of SARS-CoV-2 appeared to have no underlying health conditions, similar to $24 \%$ ( 729 of the 3,080 CYP) who died of all other causes. These six deaths included two CYP who died of PIMS-TS.

Neurological conditions were the most common comorbidity in both the CYP who died of SARS-CoV-2 $(n=13 / 25,52 \%)$ 
10,000

1,000

100

10

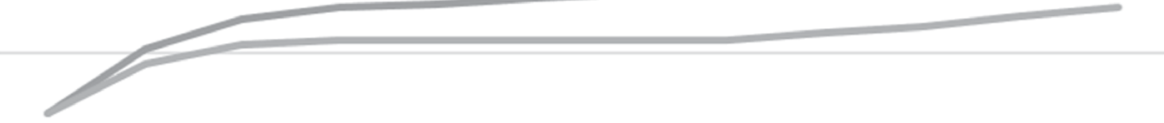

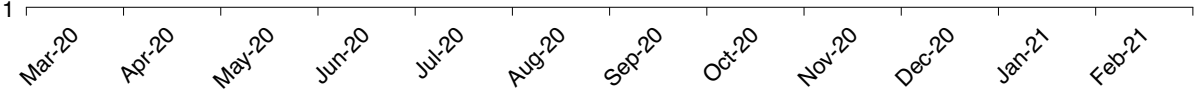

Deaths in children and young people with positive SARS-CoV-2 test

Deaths in children and young people who died of SARS-CoV-2

Fig. 2 | Graph showing the cumulative number of deaths for all CYP who died with a positive SARS-CoV-2 test in the study period (March 2020February 2021). This graph compares the number of CYP who died of SARS-CoV-2, the number of CYP who died with a positive SARS-CoV-2 test and the number of CYP who died of all other causes.

and the CYP who died of all other causes $(n=1,218 / 3,080,40 \%)$ (chi-squared 1.6, $P=0.29$ ). The chronic disease coding list used to identify neurological conditions included mental-health-related and learning-disability-related codes. All 13 CYP who died of SARS-COV-2 with a neurological comorbidity had a complex neurodisability due to a combination of an underlying genetic or metabolic condition, hypoxic ischemic events or prematurity. Eight (32\%) of the 13 CYP who had a neurological comorbidity also had a respiratory comorbidity, including five who required home respiratory support, four with non-invasive ventilation or high-flow oxygen and one with low-flow oxygen. There were zero CYP who died of SARS-CoV-2 who were invasively home ventilated. There was one death in a young person with a tracheostomy required for airway patency.

Of the 25 CYP who died of SARS-CoV-2, there was one child with each of the following comorbidities: congenital cardiac, oncological, obesity (under endocrinology) and complications of prematurity. There were two CYP who died with a hematological comorbidity.

There were no deaths in CYP with the following conditions:

- An isolated respiratory condition-for example, cystic fibrosis or asthma (three of the CYP with a complex neurodisability had a historic diagnosis of asthma; however, the asthma diagnosis was not considered to contribute to death).

- Type 1 diabetes

- Trisomy 21

- Isolated diagnosis of epilepsy

- A mental health disorder that caused or contributed to death

There were CYP with asthma and epilepsy who died of SARS-CoV-2 infection. However, all of these deaths occurred in CYP with other underlying health conditions, rather than as a single diagnosis (Fig. 3).

The estimated mortality rate for CYP who died of SARS-CoV-2 with a life-limiting condition was 11.5 per 100,000 (95\% CI, 5.6$21.2)$ compared to 1,124 per 100,000 (95\% CI, $1,054-1,197)$ for all other causes of death. Although the proportion of CYP with a life-limiting neurodisability who died of SARS-CoV-2 was higher, their absolute risk of death was 88.9 per 100,000 (95\% CI, 47.3-152) compared to 2,441 per 100,000 (95\% CI, 2,194-2,707) in CYP with a life-limiting neurodisability who died of all other causes.

Place of death. Nine (36\%) of the 25 CYP who died of SARS-CoV-2 died in a pediatric intensive care unit, and four died in a hospital ward. The remaining $12 \mathrm{CYP}$ died either at home (unexpected $(n=6)$ or expected $(n=2))$ or in an emergency department $(n=4)$. There were five deaths in CYP with advance care plans in place to provide hospital-ward-level care rather than escalation to intensive care.

Time interval between positive SARS-CoV-2 test and death. Twenty-three CYP died of SARS-CoV-2 within $28 \mathrm{~d}$ of a positive SARS-CoV-2 test; of these deaths, 21 occurred within $7 \mathrm{~d}$ of a positive test. The maximum time between death and a positive SARS-CoV-2 test was $45 \mathrm{~d}$.

The 3,044 CYP who died and did not have a positive SARS-CoV-2 test would have only had a SARS-CoV-2 test in the following circumstances: asymptomatic lateral flow tests performed for education or social activities (note that this is highly variable); symptoms consistent with acute SARS-CoV-2 infection; hospital admission; and unexpected death or postmortem examination. Therefore, not all of the 3,044 CYP who died from other causes would have been tested for SARS-CoV-2. However, none of them had a positive SARS-CoV-2 test, because we included all CYP who tested positive at any time point during the pandemic $(n=61)$, and zero positive tests were excluded from the study.

\section{Discussion}

We used a high-quality, unique national mortality dataset linked to national hospital and SARS-CoV-2 Public Health England testing data, in conjunction with clinical review, to identify 25 CYP who died of SARS-CoV-2 infection during the first pandemic year. This corresponds to two deaths per million across the CYP population in England. We estimated the infection fatality rate to be five per 100,000 , indicating that more than $99.995 \%$ of CYP recover from SARS-CoV-2 infection. SARS-CoV-2 contributed to $0.8 \%$ of the 3,105 deaths from all causes. During the same time period studied, there were 124 deaths from suicide and 268 deaths from trauma, emphasizing that COVID-19 is rarely fatal in CYP. 
Table 2 | Comorbidity details for CYP who died between March 2020 and February 2021 from all causes and the 61 CYP who died with a positive SARS-CoV-2 test, split by the likely cause of death

All deaths, March 2020-February $2021(n=3,105)$

\begin{tabular}{|c|c|c|c|c|c|c|c|}
\hline \multirow[t]{3}{*}{ All deaths } & \multicolumn{4}{|c|}{ Reference population $(n=3,080)$} & \multirow{2}{*}{\multicolumn{2}{|c|}{$\begin{array}{l}\text { Died of COVID-19/ } \\
\text { PIMS-TS }(n=25)\end{array}$}} & \multirow{3}{*}{$\begin{array}{l}\text { Comparison of } \\
\text { frequencies }^{\mathrm{a}} \\
P\end{array}$} \\
\hline & \multicolumn{2}{|c|}{ All causes $(n=3,044)$} & \multicolumn{2}{|c|}{$\begin{array}{c}\text { Incidental positive SARS-CoV-2 } \\
\text { test at death }(n=36)\end{array}$} & & & \\
\hline & Number & Percentage & Number & Percentage & Number & Percentage & \\
\hline \multicolumn{8}{|l|}{ Life-limiting condition } \\
\hline Yes & 974 & $32.0 \%$ & 14 & $38.9 \%$ & 15 & $60 \cdot 0 \%$ & 0.005 \\
\hline No & 2,027 & $66.6 \%$ & 22 & $61 \cdot 1 \%$ & 10 & $40 \cdot 0 \%$ & \\
\hline Yes & 2,238 & $73.5 \%$ & 29 & $80.6 \%$ & 19 & $76.0 \%$ & 0.6 \\
\hline No & 716 & $23.5 \%$ & 7 & $19.4 \%$ & 6 & $24.0 \%$ & \\
\hline \multicolumn{8}{|l|}{ Chronic condition details } \\
\hline Cardiology (non-congenital) & 458 & $15.0 \%$ & 8 & $22.2 \%$ & 8 & $32.0 \%$ & 0.02 \\
\hline Cardiology (congenital) & 667 & $21.9 \%$ & 10 & $27.8 \%$ & 1 & $4.0 \%$ & 0.03 \\
\hline Gastroenterology & 467 & $15.3 \%$ & 18 & $50.0 \%$ & 5 & $20.0 \%$ & 0.56 \\
\hline Genetic & 88 & $2.9 \%$ & 1 & $2.8 \%$ & 8 & $32.0 \%$ & $<0.001$ \\
\hline Hematological & 287 & $9.4 \%$ & 12 & $33.3 \%$ & 2 & $8.0 \%$ & 0.81 \\
\hline Immunological & 19 & $0.6 \%$ & 2 & $5.6 \%$ & 1 & $4.0 \%$ & 0.16 \\
\hline Infectious disease & 15 & $0.5 \%$ & 0 & $0.0 \%$ & 0 & $0.0 \%$ & - \\
\hline Metabolic & 181 & $5.9 \%$ & 7 & $19.4 \%$ & 4 & $16.0 \%$ & 0.07 \\
\hline Musculoskeletal & 142 & $4.7 \%$ & 5 & $13.9 \%$ & 4 & $16.0 \%$ & 0.03 \\
\hline Neurological & 1,194 & $39.2 \%$ & 24 & $66.7 \%$ & 13 & $52.0 \%$ & 0.29 \\
\hline Oncology & 190 & $6.2 \%$ & 8 & $22.2 \%$ & 1 & $4.0 \%$ & 0.51 \\
\hline 0 & 716 & $23.5 \%$ & 13 & $21.3 \%$ & 6 & $24.0 \%$ & 0.14 \\
\hline 1 & 906 & $29.8 \%$ & 7 & $11.5 \%$ & 3 & $12.0 \%$ & \\
\hline 2 or more & 1,332 & $43.8 \%$ & 41 & $67.2 \%$ & 16 & $64.0 \%$ & \\
\hline Unknown & 90 & $3.0 \%$ & 0 & $0.0 \%$ & 0 & $0.0 \%$ & \\
\hline Total & 3,044 & $100.0 \%$ & 61 & $100.0 \%$ & 25 & $100.0 \%$ & - \\
\hline \multicolumn{8}{|l|}{ Comorbidity combinations } \\
\hline Neurological and respiratory & 318 & $10.4 \%$ & 17 & $27.9 \%$ & 8 & $32.0 \%$ & $<0.001$ \\
\hline Neurological and cardiology & 559 & $18.4 \%$ & 15 & $24.6 \%$ & 3 & $12.0 \%$ & 0.61 \\
\hline Respiratory and cardiology & 270 & $8.9 \%$ & 12 & $19.7 \%$ & 3 & $12.0 \%$ & 0.49 \\
\hline \multicolumn{8}{|l|}{ Single diagnoses } \\
\hline Asthmab & 58 & $1.9 \%$ & 5 & $8.2 \%$ & 3 & $12.0 \%$ & 0.02 \\
\hline Type 1 diabetes & 9 & $0.3 \%$ & 0 & $0.0 \%$ & 0 & $0.0 \%$ & - \\
\hline Epilepsy ${ }^{b}$ & 199 & $6.5 \%$ & 7 & $11.5 \%$ & 7 & $28.0 \%$ & $<0.001$ \\
\hline Sickle cell disease & 1 & $0.0 \%$ & 1 & $1.6 \%$ & 1 & $4.0 \%$ & 0.02 \\
\hline Trisomy 21 & 38 & $1.2 \%$ & 0 & $0.0 \%$ & 0 & $0.0 \%$ & - \\
\hline
\end{tabular}

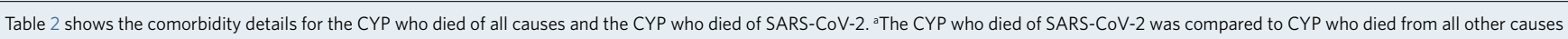
using summary statistics, and differences between groups were compared using the two-sided chi-squared test or Fisher's exact test if small numbers. No adjustment for multiple testing was undertaken.

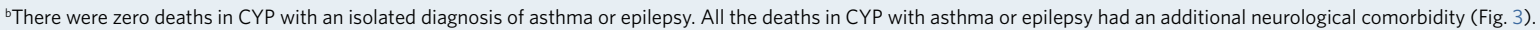


Table 3 | Estimated mortality rates by selected diagnostic groups for CYP who died of SARS-CoV-2 and CYP who died of all other causes

\begin{tabular}{|c|c|c|c|c|c|}
\hline & $\begin{array}{l}\text { Estimated } \\
\text { population at risk }\end{array}$ & \multicolumn{2}{|c|}{$\begin{array}{l}\text { Reference population, all other causes } \\
\qquad(n=3,080)\end{array}$} & \multicolumn{2}{|c|}{ Died of COVID-19/PIMS-TS $(n=25)$} \\
\hline Oncology & 1,065 (ref. ${ }^{23}$ ) & 137 & $12,864(10,800-15,207)$ & 1 & $93.9(2.4-523.2)$ \\
\hline Life-limiting neurodisability & 14,626 (ref. ${ }^{18}$ ) & 357 & $2,441(2,194-2,707)$ & 13 & $88.9(47.3-152.0)$ \\
\hline Epilepsya $^{a}$ & $90,000\left(\right.$ ref. $\left.^{25}\right)$ & 199 & $214(185-246)$ & 7 & $7.5(3.0-15.5)$ \\
\hline Asthma ${ }^{a}$ & $1,100,000$ (ref. ${ }^{26}$ ) & 58 & $5.3(4.0-6.8)$ & 3 & $0.3(0.06-0.8)$ \\
\hline
\end{tabular}

Table 3 shows the mortality rate for all CYP and for all deaths within selected diagnostic groups: Oncology, life-limiting neurodisability and life-limiting condition; Cardiology (congenital); Epilepsy; and

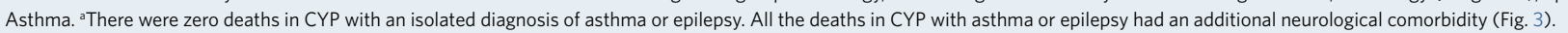

To our knowledge, this is the first study to differentiate between CYP who died of SARS-CoV-2 infection and CYP who died with a positive SARS-CoV-2 test as a coincidental finding. Our result is $60 \%$ lower than the figures derived from positive tests, thereby markedly reducing the estimated number of CYP who are potentially at risk of death during this pandemic ${ }^{14}$.

The CYP who died of SARS-CoV-2 were more likely to be teenagers than younger children, suggesting a continuum of risk increasing through the life course from infancy to older adult life ${ }^{15}$. Higher proportions of Asian and Black CYP died of SARS-CoV-2 compared to all other causes of death, although deaths were still extremely rare. The three CYP who died of PIMS-TS were all aged 10-14 years, two were male, all were from different ethnic groups and two did not have evidence of an underlying health condition.

The reason for ethnic differences might be due to biological predisposition and/or access to care. Of note, the differences persist when controlling for deprivation ${ }^{16}$. These findings support those found in adult studies ${ }^{15,17}$.

Our findings emphasize the importance of underlying comorbidities as the main risk factor for death, as $76 \%$ had chronic conditions, $64 \%$ had multiple comorbidities and $60 \%$ had life-limiting conditions. The comorbidity group at highest risk included CYP with complex neurodisability, which comprised $52 \%$ of all deaths in CYP who died of SARS-CoV-2. CYP with combined neurodisability and respiratory conditions (eight of the 13 deaths with neurodisability) might be at particularly high risk. CYP with a life-limiting neurodisability have a higher background mortality rate than the general population ${ }^{18}$. There are approximately 500 deaths annually in this group, and, therefore, SARS-CoV-2 contributed to only $3 \%$ during the pandemic. Similarly, for all other comorbidity groups, those who died of SARS-CoV-2 represented a very small proportion of all deaths during the pandemic year. It is important to note that we observed no deaths in groups that have been considered at higher risk of respiratory infections, such as CYP with asthma, cystic fibrosis, type 1 diabetes or trisomy 21 .

The inclusion of trauma as a chronic condition relates to the broad definition of chronic conditions used in this work to ensure optimal capture. The chronic condition definition (Supplementary Information) includes any health problem requiring follow-up services in more than $50 \%$ of cases, and follow-up includes use of support services such as physiotherapy. There is a subcategory of skeletal injuries/amputations that accounts for these trauma codes that are historic but chronic in nature. The high number of CYP with ear, nose and throat (ENT) conditions is due to a high proportion of CYP with neurological and/or respiratory conditions having
ENT conditions and does not relate to CYP with isolated ENT conditions. It also includes CYP with a tracheostomy.

Six CYP who died of SARS-CoV-2 had no evidence of an underlying health condition. This contrasts with other studies that have only reported deaths in CYP who have comorbidity ${ }^{7,19}$. It is possible, due to the hospital data being available only for the last 5 years, that some CYP might have had a comorbidity that was not identified in this linkage. It is also possible that CYP in our study had an undiagnosed genetic predisposition to severe disease with SARS-CoV-2 infection ${ }^{20}$.

Our findings extend previous more limited reports on deaths due to SARS-CoV-2 in the United Kingdom ${ }^{7,8,19}$. The International Severe Acute Respiratory and Emerging Infection Consortium study reported six deaths from 651 admissions across 138 hospitals up to July 2020 (ref. ${ }^{19}$ ). All six CYP had 'profound comorbidity', which included neurodisability, extreme prematurity, malignancy and sepsis; three were infants under $28 \mathrm{~d}$ of age; and three were aged $15-18$ years $^{19}$. The methodology in our study enabled demonstration that zero neonates died of SARS-CoV-2, highlighting the value of having real-time, complete mortality surveillance for CYP, with linkage to virology data and the detailed clinical review that we undertook to determine the role of SARS-CoV-2 in death.

The current United Kingdom advice on those defined as 'clinically extremely vulnerable' was initially extrapolated from adult risk, and it remains very cautious ${ }^{14}$. Even taking into consideration the effect of shielding (as both adults and CYP shielded at times during this period), the risk of serious outcomes from SARS-CoV-2 for individuals under 18 years of age remains extremely low. The risk of removal of CYP from their normal activities across education and social events might prove a greater risk than that of SARS-CoV-2 itself ${ }^{21}$.

Limitations. The SARS-CoV-2 virus strains circulating at the time of this review were wild-type and the Alpha variant from November 2020. These data are specific to the time period studied and before the advent of the Delta variant.

The data analyzed in this study largely relied upon the quality of the data entered through the NCMD death reporting process. Data completeness was variable, depending on stage of the child death review process. Where possible, we overcame this through discussion with reporting clinicians and data linkage. Rapid data linkage methods were undertaken using NHS number alone, so this might have resulted in some CYP not being matched to their hospital data.

Eight of the CYP who died of SARS-CoV-2 had a non-congenital cardiac condition recorded, despite our attempts to modify the International Classification of Diseases (ICD)-10 coding lists to account for this. Owing to the complexity of these cases, some of 


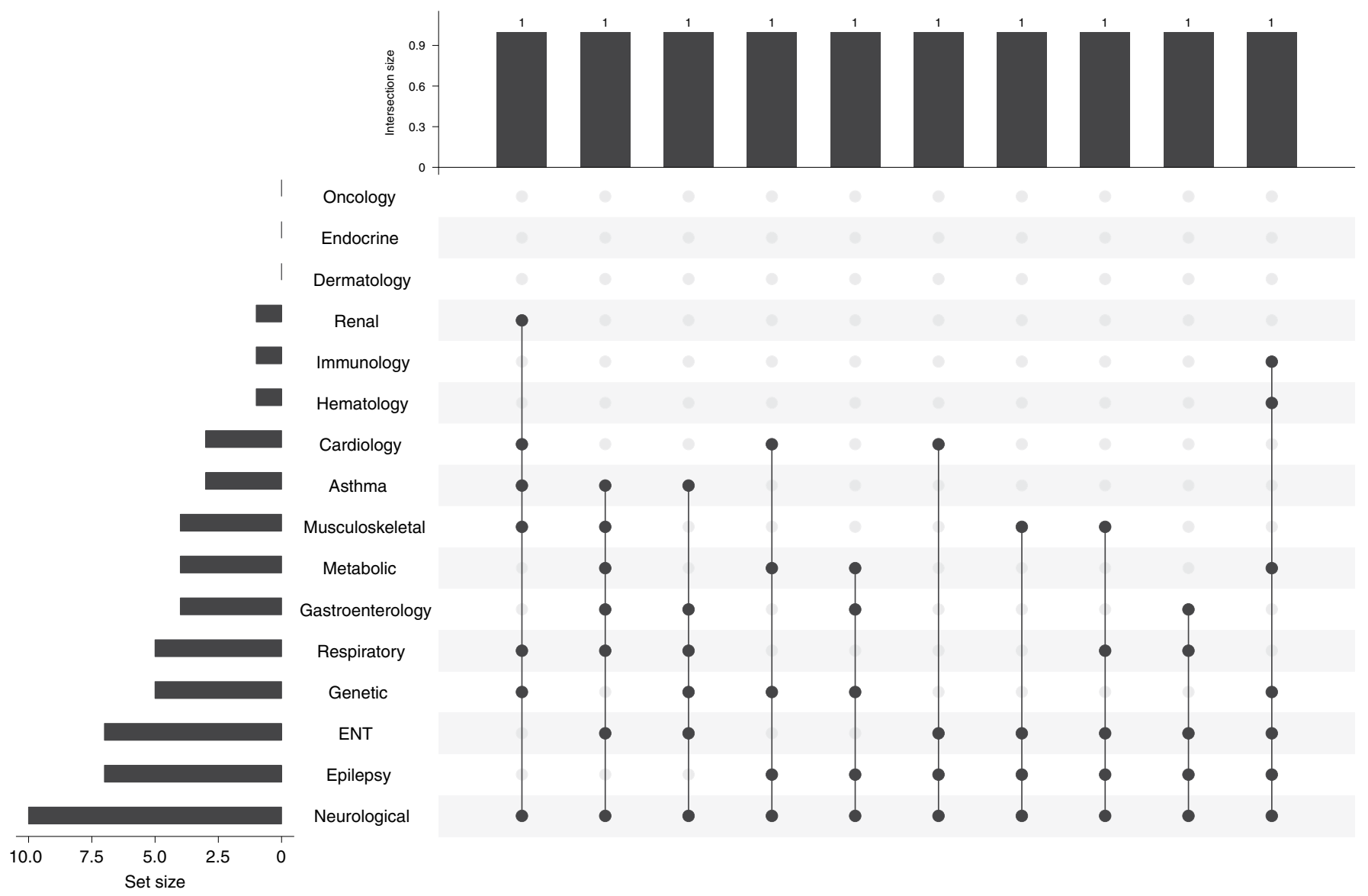

Fig. 3 | Upset plot to visualize the intersections between the single diagnosis codes of asthma and epilepsy. For individual CYP with epilepsy or asthma, the plot highlights their other comorbidities with a black circle, showing that these single diagnosis codes did not occur in isolation.

these conditions might have been as a result of COVID-19 rather than pre-existing chronic conditions.

Even though we undertook rigorous clinical review, there might still have been a potential for misclassification of deaths in this study. All sudden and unexpected deaths were tested for SARS-CoV-2 as part of the amended Joint Agency Response policy from March 2020 (ref. ${ }^{22}$ ). However, not all community deaths will have been routinely tested.

As there is no diagnostic test for PIMS-TS, and coding was a challenge, it is possible that there might be omissions due to the methods of diagnosis and reporting.

The mortality rate calculations used data from the Office for National Statistics for the estimated number of children by age in England during mid-2019 (ref. ${ }^{13}$ ). There is a paucity of accurate data on the number of children who have had SARS-CoV-2 testing, affecting the accuracy of the infection fatality rate calculation.

Going forward, linkage of the NCMD to other national datasets will enable complete capture of comorbidities in CYP. These findings are representative of the wild-type and Alpha SARS-CoV-2 variants that were prevalent at the time of the study. It would be beneficial to repeat this for the subsequent 12 months (March 2021February 2022) to identify the effect of other variants (including Delta) and vaccination.

\section{Online content}

Any methods, additional references, Nature Research reporting summaries, source data, extended data, supplementary information, acknowledgements, peer review information; details of author contributions and competing interests; and statements of data and code availability are available at https://doi.org/10.1038/s41591021-01578-1.

Received: 20 July 2021; Accepted: 13 October 2021; Published online: 11 November 2021

\section{References}

1. Viner, R. et al. Systematic review of reviews of symptoms and signs of COVID-19 in children and adolescents. Arch. Dis. Child. https://doi. org/10.1136/archdischild-2020-320972

2. Docherty, A. B. et al. Features of $20133 \mathrm{UK}$ patients in hospital with COVID-19 using the ISARIC WHO Clinical Characterisation Protocol: prospective observational cohort study. Brit. Med. J. 369, m1985 (2020).

3. de Souza, T., Nadal, J., Nogueira, R., Pereira, R. \& Brandão, M. Clinical manifestations of children with COVID-19: a systematic review. Pediatr. Pulmonol. 55, 1892-1899 (2020).

4. Bhopal, S. S., Bagaria, J., Olabi, B. \& Bhopal, R. CYP remain at low risk of COVID-19 mortality. Lancet Child Adolesc. Health 5, e12-e13 (2021).

5. Davies, P. et al. Intensive care admissions of children with paediatric inflammatory multisystem syndrome temporally associated with SARS-CoV-2 (PIMS-TS) in the UK: a multicentre observational study. Lancet Child Adolesc. Health 4, 669-77. (2020).

6. Whittaker, E. et al. Clinical characteristics of 58 children with a pediatric inflammatory multisystem syndrome temporally associated with SARS-CoV-2. JAMA 324, 259-269 (2020).

7. Flood, J. et al. Paediatric multisystem inflammatory syndrome temporally associated with SARS-CoV-2 (PIMS-TS): prospective, national surveillance, United Kingdom and Ireland. Lancet Reg. Health Eur. 3, 1-11 (2020). 100075.

8. Odd, D. et al. Child mortality in England during the COVID-19 pandemic. Arch. Dis. Child. 0, 1-7 (2021).

9. Deaths involving coronavirus disease 2019 (COVID-19) with a focus on ages 0-18 in the United States. https://data.cdc.gov/NCHS/Provisional-COVID19-Deaths-Focus-on-Ages-0-18-Yea/nr4s-juj3 
10. Prevalence of SARS-CoV-2 in children and young people in England. Data from the Office for National Statistics. https://www.ons.gov.uk/ peoplepopulationandcommunity/healthandsocialcare/conditionsanddiseases/ bulletins/coronaviruscovid19infectionsurveypilot/11june2021

11. National Child Mortality Database annual report. https://www.ncmd.info/ wp-content/uploads/2020/11/Main-Text-FINAL-WEB.pdf

12. Guidance for doctors completing medical certificates in England. https:// assets.publishing.service.gov.uk/government/uploads/system/uploads/ attachment_data/file/877302/guidance-for-doctors-completing-medicalcertificates-of-cause-of-death-covid-19.pdf

13. ONS data for estimated number of children by age living in England, mid 2019 estimate https://www.ons.gov.uk/peoplepopulationandcommunity/ populationandmigration/populationestimates

14. Royal College of Paediatrics and Child Health. COVID-19-guidance on clinically extremely vulnerable children and young people. 2021. https://www. rcpch.ac.uk/resources/covid-19-guidance-clinically-extremely-vulnerablechildren-young-people

15. Williamson, E. J. et al. Factors associated with COVID-19-related death using OpenSAFELY. Nature 584, 430-436 (2020).

16. Parslow, R. C. et al. Epidemiology of critically ill children in England and Wales: incidence, mortality, deprivation and ethnicity. Arch. Dis. Child. 94, 210-215 (2009).

17. Clift, A. K. et al. Living risk prediction algorithm (QCOVID) for risk of hospital admission and mortality from coronavirus 19 in adults: national derivation and validation cohort study. Brit. Med. J. 371, m3731 (2020).

18. 'Make Every Child Count'. Estimating current and future prevalence of CYP with life-limiting conditions in the United Kingdom. https://www. togetherforshortlives.org.uk/resource/make-every-child-count/

19. Swann, O. V. et al. Clinical characteristics of CYP admitted to hospital with COVID-19 in United Kingdom: prospective multicentre observational cohort study. Brit. Med. J. 370, m3249 (2020).
20. Anastassopoulou, C. et al. Human genetic factors associated with susceptibility to SARS-CoV-2 infection and COVID-19 disease severity. Hum. Genomics 14, 40 (2020).

21. Petretto, D. R., Masala, I. \& Masala, C. School closure and children in the outbreak of COVID-19. Clin. Pract. Epidemiol. Ment. Health 16, 189-191 (2020).

22. NCMD contributions to modifying the investigation protocol for sudden unexpected deaths in CYP to include post-mortem testing for SARS-CoV-2. https://www.ncmd.info/2020/04/07/jar-covid-19/

23. Children and young people receiving treatment for an oncological condition in England. Data obtained from SUS data (see reference 12) and discussed with Cancer Programme of Care - Specialised Commissioning, NHS England and NHS Improvement. https://www.england.nhs.uk/commissioning/ spec-services/npc-crg/group-b/ (data provided 17 June 2021).

24. Children and young people with congenital heart disease in England. Data collected as part of National Institute for Cardiovascular Outcomes Research (NICOR). Data provided by Clinical Reference Group Congenital Heart Disease, NHS England. https://www.england.nhs.uk/commissioning/ spec-services/npc-crg/group-e/e05/

25. Children and young people with a diagnosis of epilepsy in England. https:// www.england.nhs.uk/wp-content/uploads/2018/09/E09-S-b-PaediatricNeurosciences-Neurology.pro_.2013.04.v2.pdf

26. Children and young people with a diagnosis of asthma in England. Estimates provided by the National Asthma and COPD Audit Programme (NACAP), Royal College of Physicians, England. https://www. nacap.org.uk/

Publisher's note Springer Nature remains neutral with regard to jurisdictional claims in published maps and institutional affiliations.

(C) The Author(s), under exclusive licence to Springer Nature America, Inc. 2021 


\section{Methods}

Population. The cohort investigated in this study is all CYP under 18 years of age who died in England between 1 March 2020 and 28 February 2021 (ref. ${ }^{11}$ ). The aim of this study was to identify CYP in which SARS-CoV-2 contributed to death-that is, they died of SARS-CoV-2 infection.

Ethics approval was granted by the Central Bristol NHS Research and Ethics Committee. Informed consent was not obtained for use of these data. The NCMD has a legal basis to collect data without consent (Supplementary Information) ${ }^{27}$. Current Control Of Patient Information regulations provide a legal basis for linking NCMD data with Secondary Uses Service data without consent ${ }^{28}$. Additional details are provided in the Supplementary Information.

A statistical risk assessment for this study determined that, although data are anonymized, identification of individuals might be possible. However, the risk of attribute details being disclosed was low, and the public benefit of reporting these small numbers outweighed this risk. We minimized this risk by providing data that are two dimensional rather than three dimensional-for example, we provided the number of CYP in each age or ethnicity category rather than providing linked comorbidity and demographic details for each CYP. Given the sensitive nature of these data and our awareness that clinicians and families might recognize personal experience, we met with a clinician or professional involved in the care of each child or young person who died of SARS-CoV-2 infection. We asked the respective clinician or professional to communicate this work directly to the families.

Data collection. The NCMD is a mandatory system that records all deaths in CYP under 18 years of age in England since it began in April 2019 (ref. ${ }^{11}$ ) and includes demographic and clinical data of the events leading up to death.

In this analysis, demographic details included age (coded as 0-27 d, 28-364 d, $1-4$ years, 5-9 years, $10-14$ years and $15-17$ years), sex, ethnicity (coded as Asian or Asian British, Black or African or Caribbean or Black British, mixed, multiple, other (includes Arab and other ethnic groups) and white ${ }^{29}$ and deprivation (Supplementary Information) $)^{30,31}$.

Data linkage. To ensure comprehensive identification of comorbidities, NCMD data were linked to the preceding 5 years (March 2015 onwards) of national admitted patient care Secondary Uses Service data for England ${ }^{32}$ and to the national Paediatric Intensive Care Audit Network data. A validated list of ICD-10 codes was used to identify CYP with chronic comorbidities ${ }^{33}$ and life-limiting conditions ${ }^{34}$ (Supplementary Information). Of note, the chronic disease list for cardiac conditions was modified to remove 'I46-Cardiac Arrest' and 'I51-Complications and ill-defined descriptions of heart disease, as these are acute presentations of cardiac disease and likely to represent PIMS-TS rather than pre-existing comorbidity. We also identified CYP with chronic comorbidities in two or more body systems and with the following single diagnoses: asthma, diabetes, epilepsy, sickle cell disease and trisomy 21 . These single diagnoses were identified as common long-term conditions in CYP and speculated to be risk factors for severe disease based on adult data (refs. ${ }^{15,17}$ ).

SARS-CoV-2 data. During the pandemic, the NCMD was linked by NHS number to Public Health England Pillar 1 and Pillar 2 testing data ${ }^{35}$ to identify all CYP who died with a positive SARS-CoV-2 test. Pillar 1 testing occurs in health and care settings, whereas Pillar 2 testing occurs in the community ${ }^{35}$, both of which started in March 2020. The NCMD contributed to modification of the protocol for sudden, unexpected deaths in CYP to include postmortem testing for SARS-CoV-2 (ref. ${ }^{22}$ ). All CYP who died with a positive SARS-CoV-2 test were included, regardless of the time interval between positive test and death. This is different than the definition used for reporting adult deaths, to ensure that all potential cases were identified for review and to optimize capture of possible PIMS-TS cases. In addition, the NCMD coding team identified potential cases of PIMS-TS (Supplementary Information).

Identifying CYP who died of SARS-CoV-2. Clinical records of all CYP who died with a positive SARS-CoV-2 test were reviewed to identify if SARS-CoV-2 clearly, probably, possibly or unlikely contributed to death. This process initially included identifying whether SARS-CoV-2 was listed as 1a (the direct cause of death) on the Certificate of Cause of Death and whether the clinical course described was typical of SARS-CoV-2 infection. In these circumstances, the classification 'SARS-CoV-2 clearly contributed to death' was applied. In England, the Certificate of Cause of Death is set out in two parts ${ }^{12}$. Part la is the immediate, direct cause of death. The sequence of events or conditions that led to the death are then listed as $1 \mathrm{~b}$ and $1 \mathrm{c}$ (if necessary). Other disease, injuries, conditions or events that contributed to death but were not part of the direct sequence are then documented in Part 2 (ref. ${ }^{12}$ ).

If it was not clearly apparent, each case underwent review by three independent senior clinical experts in relevant fields (General Pediatrics, Neonatology and Pediatric Intensive Care) who were asked to classify each case. Each senior clinical expert was blinded to the opinion of the other reviewers. Definitions for each category and the details behind the process are outlined in the Supplementary Information and Fig. 1.

Statistical analysis. The CYP who died of SARS-CoV-2 were compared to CYP who died from all other causes using summary statistics, and differences between groups were compared using the two-sided chi-squared test or Fisher's exact test if small numbers. The comparator cohort, death from all other causes, included CYP who tested positive for SARS-CoV-2 but died of another cause. Owing to a small amount of missing data, multiple imputation was not undertaken.

The absolute risk of death was calculated for the whole population and for demographic groups in which denominator data were available. The quality of available data on the number of CYP in the population with comorbidities was variable. We used estimates for comorbidity groups, where we have enough confidence in the data, to derive estimated absolute risk. These data came from a range of sources and are referenced in Table 3.

The infection fatality rate was calculated using the number of CYP infected with SARS-CoV-2 during the same time period (March 2020-February 2021) estimated through Public Health England modeling data ${ }^{36}$. This was chosen rather than the absolute number of positive SARS-CoV-2 tests as CYP might test positive more than once, and many CYP were not tested in the first wave of the pandemic. Mortality rate was calculated using a population of $12,023,568$ CYP living in England ${ }^{13}$ during the study year.

This study was reported according to the 'Strengthening the Reporting of Observational Studies in Epidemiology Statement: guideline for reporting observational studies ${ }^{37}$.

Reporting Summary. Further information on research design is available in the Nature Research Reporting Summary linked to this article.

\section{Data availability}

Data used for this study are not publicly available because the information is highly sensitive, owing to it being available at the identifiable patient level because of small numbers. The analysis was performed in Microsoft Excel using basic count functions to identify CYP within each category. Statistical analyses were performed in Stata using the data in Tables 1 and 2.

\section{References}

27. National Child Mortality Database legal basis for collecting personal and confidential data. https://consult.education.gov.uk/child-protectionsafeguarding-and-family-law/working-together-to-safeguard-childrenrevisions-t/supporting_documents/Working\%20Together\%20to\%20 Safeguard\%20Children.pdf

28. Control Of Patient Information (COPI) regulations provide a legal basis for linking NCMD data with SUS data. https://digital.nhs.uk/coronavirus/ coronavirus-covid-19-response-information-governance-hub/control-ofpatient-information-copi-notice

29. Ethnicity grouping methodology. https://www.ethnicity-facts-figures.service. gov.uk/style-guide/ethnic-groups

30. Office for National Statistics. Census geography. An overview of the various geographies used in the production of statistics collected via the UK census. 2019. https://www.ons.gov.uk/methodology/geography/ukgeographies/censusg eography\#super-output-area-soa

31. National Child Mortality Database (NCMD) deprivation report. https://www. ncmd.info/2021/05/13/dep-report-2021/

32. Herbert, A., Wijlaars, L., Zylbersztejn, A., Cromwell, D. \& Hardelid, P. Data Resource Profile: Hospital Episode Statistics Admitted Patient Care (HES APC). Int. J. Epidemiol. 46, 1093-1093i (2017).

33. Hardelid, P., Dattani, N. \& Gilbert, R. Estimating the prevalence of chronic conditions in children who die in England, Scotland and Wales: a data linkage cohort study. BMJ Open 4, e005331 (2014).

34. Fraser, L. K. et al. Rising national prevalence of life-limiting conditions in children in England. Pediatrics 129, e923-e929 (2012).

35. Public Health England SARS-CoV-2 testing data information. https://www. gov.uk/government/publications/coronavirus-covid-19-testing-datamethodology/covid-19-testing-data-methodology-note

36. Public Health England modelling data for number of children and young people who have had SARS-CoV-2 infection in England. https://coronavirus. data.gov.uk/details/download

37. The Strengthening the Reporting of Observational Studies in Epidemiology (STROBE) Statement: guidelines for reporting observational studies. https:// www.equator-network.org/reporting-guidelines/strobe/

\section{Acknowledgements}

We would like to thank the three senior clinical expert reviewers who independently reviewed clinical case notes of the CYP who died with a positive SARS-CoV-2 test: P. Fleming, Professor of Infant Health and Developmental Physiology, University of Bristol; G. Rossouw, General Paediatrics and Neonates, Research Fellow, National Child Mortality Database (NCMD); and D. Alison Perry, Paediatric Intensive Care, Bristol Royal Hospital for Children. We are grateful to members of the Child Death Overview Panels for their support and expertise and all child death review professionals for submitting data and providing additional information when requested. The entire NCMD team (particularly N. Cook, S. Stoianova, V. Sleap and T. Williams) have been very helpful in providing data for linkage and supporting analysis. We thank the NHS Digital and the NHS England and NHS Improvement Children and Young People teams (particularly R. Owen, 
S. Solti and T. Watson-Koszel) for their contributions and support. We thank Public Health England's Field Service and National Child and Maternal Health Intelligence Network teams for their collaboration in establishing the real-time surveillance system on child deaths potentially related to COVID-19 and their ongoing support in the daily linkage with SARS-CoV-2 test results. We would like to acknowledge support from the National Institute of Health Research (NIHR) through the National School for Public Health Research Programme and the Applied Research Collaboration North West London. We would like to thank the Royal College of Paediatrics and Child Health for their contributions and support. Parent and public involvement is at the heart of the NCMD program. We are indebted to C. Bevan (Stillbirth and Neonatal Death Charity), T. McAlorum (Child Bereavement UK) and J. Ward (Lullaby Trust), who represent bereaved families on the NCMD program steering group, for their advice and support with setting up the real-time child mortality surveillance system at the beginning of the COVID-19 pandemic. The authors received no specific funding for this work. Three of the authors are in receipt of research funding for their broader employment. R.H. is in receipt of a funded fellowship from Kidney Research UK (grant no. TF_010_20171124). J.W. is in receipt of a Medical Research Council Fellowship (grant reference MR/R00160X/1). L.K.F. is in receipt of funding from Martin House Childrens Hospice (there is no specific grant number for this funding). R.V. is in receipt of a grant from the NIHR (grant no. NIHR202322, 'Understanding the disruption of children and young people's health and healthcare use during and after COVID-19 to inform healthcare and policy responses') These funders had no role in study design, data collection and analysis, decision to publish or preparation of the manuscript. The NCMD program, including this work, is funded by NHS England and commissioned by the Healthcare Quality Improvement Partnership as part of the National Clinical Audit and Patient Outcomes Programme.

\section{Author contributions}

Study design: C.S., D.O., R.H., J.W., M.L., M.C., D.H., S.N.L., E.D., P.J.D., S.E.K., E.W., K.L., R.V. and L.K.F. Data collection and analysis: C.S., D.O., L.K.F. and K.L. Data interpretation: C.S., D.O., E.H., J.W., M.L., M.C., D.H., S.N.L., E.D., P.J.D., S.E.K., E.W., K.L., R.V. and L.K.F. Review of underlying data: C.S., D.O. and L.F. Writing the first draft: C.S. Manuscript review and editing: C.S., D.O., R.H., J.W., M.L., M.C., D.H., S.N.L., E.D., P.J.D., S.E.K., E.W., K.L., R.V. and L.K.F.

\section{Competing interests}

The authors declare no competing financial interests.

\section{Additional information}

Extended data is available for this paper at https://doi.org/10.1038/s41591-021-01578-1.

Supplementary information The online version contains supplementary material available at https://doi.org/10.1038/s41591-021-01578-1.

Correspondence and requests for materials should be addressed to Clare Smith.

Peer review information Nature Medicine thanks Olivia Swann and the other, anonymous, reviewer(s) for their contribution to the peer review of this work. Alison Farrell and João Monteiro are the primary editors on this article and managed its editorial process and peer review in collaboration with the rest of the editorial team.

Reprints and permissions information is available at www.nature.com/reprints. 


\section{NATURE MEDICINE}

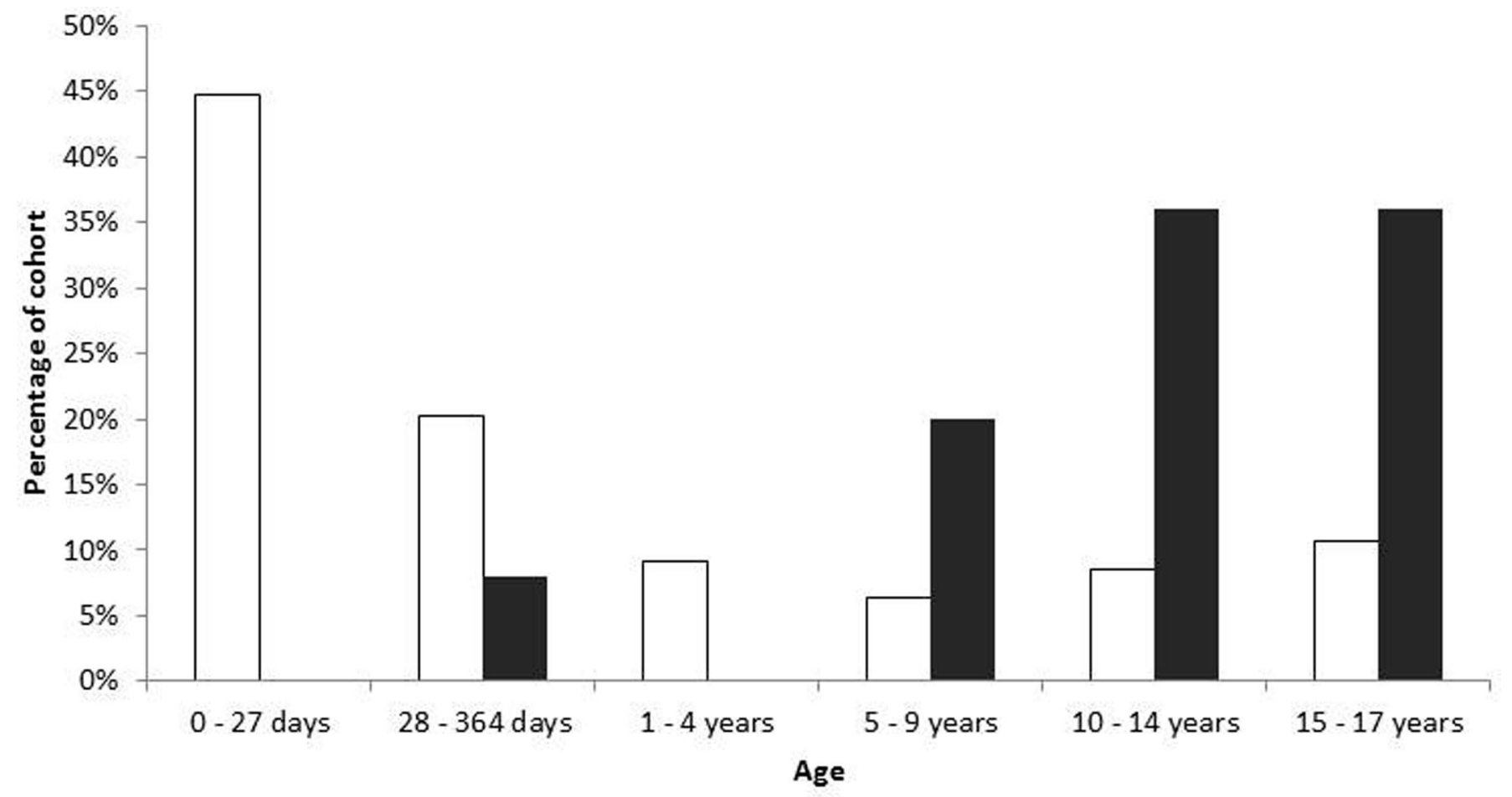

$\square$ All deaths $\square$ SARS-CoV-2 contributed to death

Extended Data Fig. 1 | Age group of the 25 Children and Young People who died of SARS-CoV-2. is a bar chart demonstrating the age group of Children and Young People (CYP) who died of SARS-CoV-2 $(n=25)$ compared to the age group of CYP deaths from all other causes $(n=3080)$. 


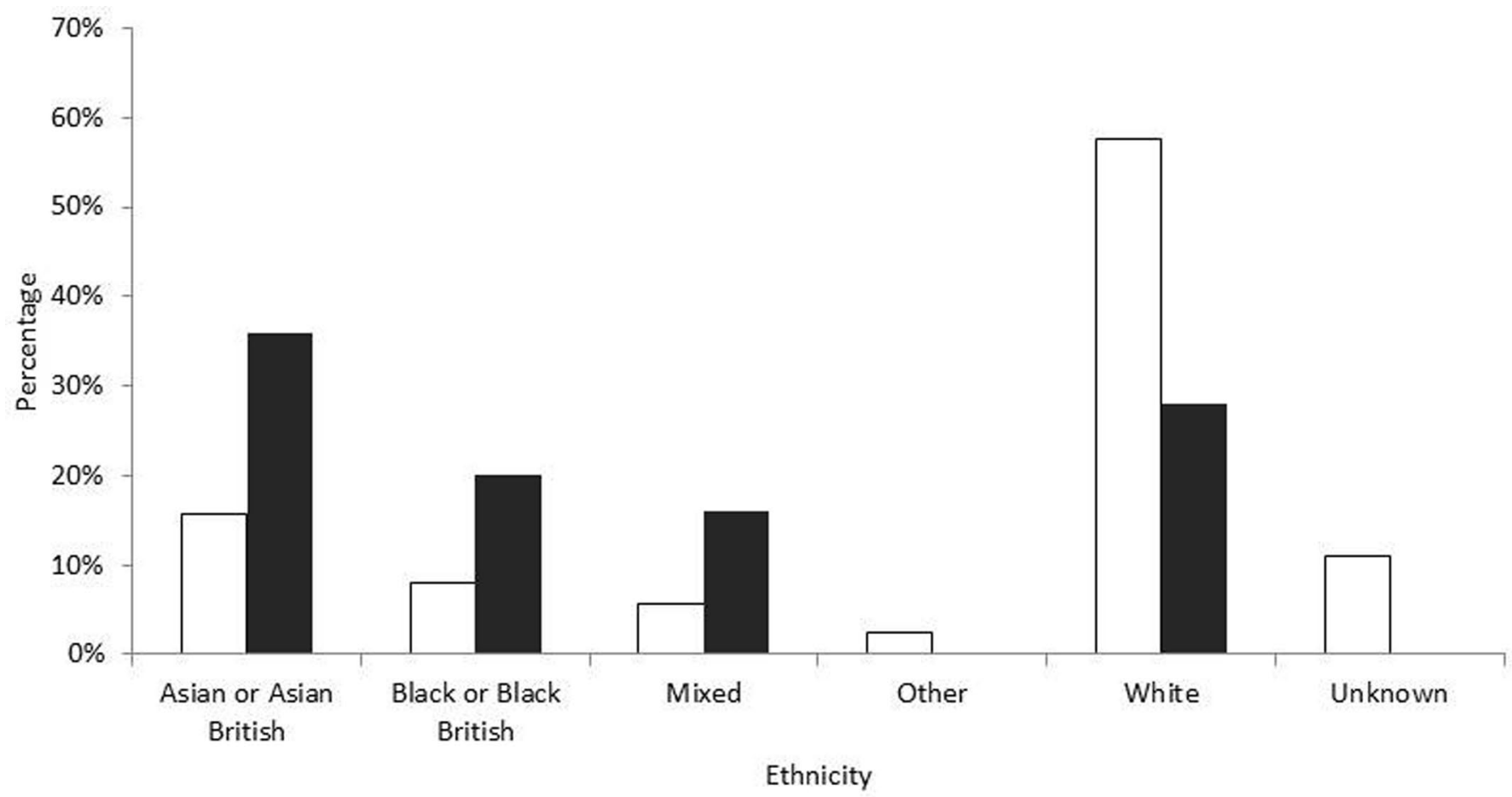

\section{$\square$ All deaths $\quad$ SARS-CoV-2 contributed to death}

Extended Data Fig. 2 | Ethnic group of the 25 Children and Young People who died of SARS-CoV-2. is a bar chat demonstrating the ethnic group of Children and Young People (CYP) who died of SARS-CoV-2 $(n=25)$ compared to the ethnic group of CYP deaths from all other causes $(n=3080)$. 


\section{Reporting Summary}

Nature Portfolio wishes to improve the reproducibility of the work that we publish. This form provides structure for consistency and transparency in reporting. For further information on Nature Portfolio policies, see our Editorial Policies and the Editorial Policy Checklist.

\section{Statistics}

For all statistical analyses, confirm that the following items are present in the figure legend, table legend, main text, or Methods section.

$\mathrm{n} / \mathrm{a} \mid$ Confirmed

$\bigotimes$ The exact sample size $(n)$ for each experimental group/condition, given as a discrete number and unit of measurement

$\square$ A statement on whether measurements were taken from distinct samples or whether the same sample was measured repeatedly

The statistical test(s) used AND whether they are one- or two-sided

Only common tests should be described solely by name; describe more complex techniques in the Methods section.

$\bigotimes$ A description of all covariates tested

Х $\square$ A description of any assumptions or corrections, such as tests of normality and adjustment for multiple comparisons

$\triangle$ A full description of the statistical parameters including central tendency (e.g. means) or other basic estimates (e.g. regression coefficient) AND variation (e.g. standard deviation) or associated estimates of uncertainty (e.g. confidence intervals)

$\triangle$ For null hypothesis testing, the test statistic (e.g. $F, t, r$ ) with confidence intervals, effect sizes, degrees of freedom and $P$ value noted

$\triangle \square$ Give $P$ values as exact values whenever suitable.

Х $\square$ For Bayesian analysis, information on the choice of priors and Markov chain Monte Carlo settings

Х $\square$ For hierarchical and complex designs, identification of the appropriate level for tests and full reporting of outcomes

$\triangle \square$ Estimates of effect sizes (e.g. Cohen's d, Pearson's $r$ ), indicating how they were calculated

\section{Our web collection on statistics for biologists contains articles on many of the points above.}

\section{Software and code}

Policy information about availability of computer code

Data collection No data software was used in data collection. Data were extracted from the National Child Mortality Database and analyzed directly from the database. Data linkage between the National Child Mortality data base and the preceding five years of national admitted patient care Secondary Uses Service (SUS) and the national Paediatric Intensive Care Audit Network (PICANet) data occurred within a National Clinical Data Repository held within NHS England. These data were then anonymized and released for analysis within Microsoft Excel.

Data analysis Data were analysed using Stata (StatCorp, USA).

For manuscripts utilizing custom algorithms or software that are central to the research but not yet described in published literature, software must be made available to editors and

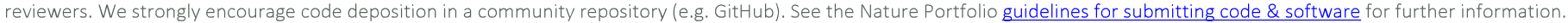

\section{Data}

Policy information about availability of data

All manuscripts must include a data availability statement. This statement should provide the following information, where applicable:

- Accession codes, unique identifiers, or web links for publicly available datasets

- A description of any restrictions on data availability

- For clinical datasets or third party data, please ensure that the statement adheres to our policy

The National Child Mortality Database is a mandatory system that records all deaths in children and young people under 18 years of age in England since it began in April 2019 and includes demographic and clinical data of the events leading up to death.

This National Child Mortality Database legal basis to collect confidential and personal level data under the Common Law Duty of Confidentiality has been established 


\section{Field-specific reporting}

Please select the one below that is the best fit for your research. If you are not sure, read the appropriate sections before making your selection.

$\bigotimes$ Life sciences

Behavioural \& social sciences

Ecological, evolutionary \& environmental sciences

For a reference copy of the document with all sections, see nature.com/documents/nr-reporting-summary-flat.pdf

\section{Life sciences study design}

All studies must disclose on these points even when the disclosure is negative.

Sample size Sample size was determined within an allocated time frame; one year between February 2020 - March 2021. This was chosen to capture the number of children and young people who died of SARS-CoV-2 within the first year of the COVID-19 pandemic in England.

Data exclusions No data were excluded from the study. We included all children and young people who died with a previous positive SARS-CoV-2 test within the defined time period. We compared this to all other deaths from all other causes during the first pandemic year.

Replication We reviewed each death for all children and young people under 18 years of age who died with a positive SARS-CoV-2 test to identify if SARSCoV-2 contributed to death or if the child/young person died of an alternative cause with a coincidental positive test. Each of the 61 cases were reviewed to identify if SARS-CoV-2 clearly contributed to death as defined by the certification of death or clear clinical course. For all other cases three senior clinical experts reviewed each case independently to provide their opinion if SARS-CoV-2 contributed to death. The classification groupings used were: SARS-CoV-2 clearly implicated in death, SARS-CoV-2 probably contributed to death, SARS-CoV-2 possibly contributed to death, SARS-CoV-2 unlikely contributed to death, Unable to classify based on the available information. If two or three senior specialist doctors agreed on the same classification then consensus was achieved. If all three senior specialist doctors disagreed two further consultants reviewed the case. The cases of SARS-CoV-2 clearly implicated in death, SARS-CoV-2 probably contributed to death, SARS-CoV-2 possibly contributed to death were combined and categorised as SARS-CoV-2 contributed to death. The cases classified as 'unable to say based on the available information' were discussed with reporting clinicians and professionals involved in the care. Further specialist opinion was sought from Paediatric Oncologists and a Paediatric Infectious Disease expert due to a high (relative) number of cases ( 9 and 13 respectively) and a need to ensure accuracy of interpretation.

Randomization Randomization was not applicable to our study.

Blinding The three senior clinical experts considering whether SARS-CoV-2 was implicated in the death of a child were blinded to the opinion of each other. No other blinding occurred.

\section{Reporting for specific materials, systems and methods}

We require information from authors about some types of materials, experimental systems and methods used in many studies. Here, indicate whether each material, system or method listed is relevant to your study. If you are not sure if a list item applies to your research, read the appropriate section before selecting a response.

\begin{tabular}{l|l} 
Materials \& experimental syste \\
\hline n/a & Involved in the study \\
\hline & $\square$ Antibodies \\
$\square$ & $\square$ Eukaryotic cell lines \\
$\square$ & $\square$ Animals and other organisms \\
$\square$ & $\square$ Clinical data \\
$\square$ & $\square$ Dual use research of concern
\end{tabular}

\begin{tabular}{l|l}
\multicolumn{2}{l}{ Methods } \\
\hline n/a & Involved in the study \\
$\triangle$ & $\square$ ChIP-seq \\
$\square$ & $\square$ Flow cytometry \\
$\square$ & $\square$ MRI-based neuroimaging
\end{tabular}

\section{Human research participants}

\section{Policy information about studies involving human research participants}

Population characteristics

The population investigated in this study is all children and young people under 18 years of age who died in England between 1st March 2020 and 28th February 2021. In the analysis, demographic details included age (coded as 0-27 days, 28-364 days, 1-4 years, 5-9 years, 10-14 years and 15-17 years), sex, ethnicity (coded as Asian or Asian British, Black or African or Carribean or Black British, Mixed, Multiple or Other, or White) and deprivation. For deprivation, using residential address data, the Index of Multiple Deprivation (IMD) 2019 were obtained. Deprivation was derived using seven main domains (income, employment, education, health (physical and mental), crime, access to housing and services, and living environment). IMD is derived from the Lower-layer Super Output Area (LSOA) of residence. Each LSOA is a small geographical area with a minimum 
population of 1000 persons and a mean population of 1500. Each LSOA is placed in one of five categories (1 to 5) containing approximately the same number of people across England, with 1 being the most deprived and 5 being the least deprived.

In addition, co-morbidities were reviewed for the population. A validated list of ICD-10 codes was used to identify children and young people with chronic co-morbidities and life-limiting conditions (see reference in paper). We identified children and young people with chronic co-morbidities in two body systems and with the following single diagnoses: asthma, diabetes, epilepsy, sickle cell and trisomy 21.

\section{Recruitment}

Ethics oversight
Participants were not recruited as reporting to the NCMD is a mandatory legal requirement in England. All deaths were included within the time frame of the study.

Bristol NHS Research and Ethics Committee (REC)

Note that full information on the approval of the study protocol must also be provided in the manuscript. 